\title{
Dendritic cells fused with different pancreatic carcinoma cells induce different T-cell responses
}

This article was published in the following Dove Press journal:

OncoTargets and Therapy

23 January 2013

Number of times this article has been viewed

\author{
Yoshiaki Andoh ${ }^{1,2}$ \\ Naohiko Makino ${ }^{2}$ \\ Mitsunori Yamakawa' \\ 'Department of Pathological \\ Diagnostics, ${ }^{2}$ Department of \\ Gastroenterology, Yamagata University \\ School of Medicine, Yamagata, Japan
}

Background: It is unclear whether there are any differences in the induction of cytotoxic $\mathrm{T}$ lymphocytes (CTL) and CD4 ${ }^{+} \mathrm{CD} 25^{\text {high }}$ regulatory T-cells (Tregs) among dendritic cells (DCs) fused with different pancreatic carcinomas. The aim of this study was to compare the ability to induce cytotoxicity by human DCs fused with different human pancreatic carcinoma cell lines and to elucidate the causes of variable cytotoxicity among cell lines.

Methods: Monocyte-derived DCs, which were generated from peripheral blood mononuclear cells (PBMCs), were fused with carcinoma cells such as Panc-1, KP-1NL, QGP-1, and KP-3L. The induction of CTL and Tregs, and cytokine profile of PBMCs stimulated by fused DCs were evaluated.

Results: The cytotoxicity against tumor targets induced by PBMCs cocultured with DCs fused with QGP-1 (DC/QGP-1) was very low, even though PBMCs cocultured with DCs fused with other cell lines induced significant cytotoxicity against the respective tumor target. The factors causing this low cytotoxicity were subsequently investigated. DC/QGP-1 induced a significant expansion of Tregs in cocultured PBMCs compared with DC/KP-3L. The level of interleukin-10 secreted in the supernatants of PBMCs cocultured with DC/QGP-1 was increased significantly compared with that in DC/KP-3L. Downregulation of major histocompatibility complex class I expression and increased secretion of vascular endothelial growth factor were observed with QGP-1, as well as in the other cell lines.

Conclusion: The present study demonstrated that the cytotoxicity induced by DCs fused with pancreatic cancer cell lines was different between each cell line, and that the reduced cytotoxicity of DC/QGP-1 might be related to the increased secretion of interleukin-10 and the extensive induction of Tregs.

Keywords: dendritic cell, cytotoxic T lymphocyte, interleukin-10, pancreatic cancer, regulatory $\mathrm{T}$ cell

\section{Introduction}

Pancreatic and biliary cancers are relatively resistant to chemotherapy and radiation and may therefore provide an opportunity for testing the potential of immunotherapy. Clinical trials of dendritic cell (DC) vaccination for advanced pancreatic cancer patients have revealed that this therapy can stimulate an antitumoral $\mathrm{T}$ cell response and prolong the survival of refractory pancreatic cancer patients, indicating a promising treatment modality. ${ }^{1-5}$ A critical issue in optimizing DC vaccines is the choice of tumor-associated antigen for DC loading. Some tumor antigens such as mucin 1 and $\alpha$-galactosylceramide have been identified as potential targets for the immunotherapy of pancreatic carcinoma. ${ }^{6-10}$ Some reports indicated the efficacy of combined therapy with DC vaccination and chemotherapeutic agents of gemcitabine. ${ }^{11-13}$ However, immunity 
against a single antigen may be ineffective in tumors with heterogeneous cell populations and may carry a risk of inducing tumor antigen escape variants. Although a recent study has demonstrated that the generation of cytotoxic $\mathrm{T}$ lymphocytes (CTL) against three or more tumor antigens correlated with clinical response, ${ }^{14}$ for many tumors, no or only few antigenic epitopes have been identified. Several recent studies have demonstrated the efficacy of the induction of antitumor immunity through the generation of fused DCs with tumor cells. ${ }^{15-17}$ In this strategy, different tumorassociated antigens, including those that are as yet unidentified, are processed endogenously and presented by major histocompatibility complex (MHC) class I pathway in the context of costimulatory signals.

The mechanisms that mediate immune tolerance to cancer are not well understood, but recent findings have revealed them to be multifactorial, including downregulation of MHC class I molecules, loss of tumor antigens, defective death receptor signaling, and generation of immunosuppressive cells such as regulatory T cells (Tregs). ${ }^{18-22}$ Major populations of these cells are $\mathrm{CD} 4{ }^{+} \mathrm{CD} 25^{\text {high }}$ Tregs that specifically express the forkhead transcription factor, forkhead/ winged helix transcription factor gene (FOXP3) and $\mathrm{CD} 4^{+}$ type 1 Tregs that secrete high levels of interleukin-10 (IL-10). Tregs secrete the immunoregulatory cytokines, IL-10 and transforming growth factor (TGF)- $\beta 1$, and inhibit T-cell proliferation. Tregs are significantly increased in a population of peripheral blood lymphocytes and tumor-infiltrating lymphocytes in some epithelial cancers. ${ }^{19-22}$ Prostaglandin $\mathrm{E}_{2}$ $\left(\mathrm{PGE}_{2}\right)$ induces FOXP3 gene expression and Treg function in $\mathrm{CD}^{+} \mathrm{T}_{\text {cells. }}{ }^{23}$

Very few reports have evaluated the efficacy of DC vaccination using pancreatic cancer cell lines, being almost limited to Panc02. ${ }^{24-27}$ However, antitumor immunity based on DCs has not yet been compared among various pancreatic carcinoma cell lines, and it remains unclear whether there are any differences in induction of CTL and Tregs among DCs fused with different pancreatic carcinoma cells. We selected four representative human pancreatic cancer cell lines, Panc-1 as undifferentiated carcinoma, ${ }^{28}$ KP-1NL as highly metastatic adenocarcinoma, ${ }^{29}$ QGP-1 as carcinoma of islet cell, ${ }^{30}$ and $\mathrm{KP}-3 \mathrm{~L}$ as adenosquamous carcinoma. $^{31}$

The aim of this study was to compare the ability to induce cytotoxicity by human DCs fused with different human pancreatic carcinoma cell lines and to elucidate the causes of variable cytotoxicity among pancreatic carcinoma cell lines.

\section{Materials and methods \\ Reagents}

Recombinant human granulocyte macrophage colonystimulating factor (GM-CSF) and IL-4 were purchased from Primmune KK (Osaka, Japan). IL-2, lipopolysaccharide (LPS) from Escherichia coli, and fluorochrome-labeled PKH-26 and PKH-67 were purchased from Sigma-Aldrich (St Louis, MO, USA).

\section{Cell culture of pancreatic carcinoma cell lines}

A human pancreatic carcinoma cell line, Panc-1 (undifferentiated carcinoma), was obtained from the Cell Resource Center for Biomedical Research, Tohoku University (Miyagi, Japan). The human pancreatic carcinoma cell lines, KP-1NL (adenocarcinoma), QGP-1 (carcinoma of islet cell), and KP-3L (adenosquamous carcinoma) were purchased from Health Science Research Resources Bank (Osaka, Japan). Cells were maintained in $75 \mathrm{~cm}^{2}$ cell culture flask with RPMI 1640 (Life Technologies, Carlsbad, CA, USA) supplemented with $10 \%$ fetal calf serum ([FCS] Life Technologies), 100 units $/ \mathrm{mL}$ penicillin, $100 \mu \mathrm{g} / \mathrm{mL}$ streptomycin, and $50 \mu \mathrm{g} / \mathrm{mL}$ gentamycin, and were grown at $37^{\circ} \mathrm{C}$ with $5 \% \mathrm{CO}_{2}$ in a humidified atmosphere.

\section{Preparation of DCs from peripheral blood} Monocyte-derived DCs were generated as described previously. ${ }^{32}$ Briefly, peripheral blood mononuclear cells (PBMCs) were isolated from peripheral blood from four healthy volunteers by Ficoll-Hypaque gradient centrifugation and were subsequently suspended in 6-well culture plates for 1 hour at $37^{\circ} \mathrm{C}$. The nonadherent cells were removed. The adherent cells were harvested and cultured in RPMI 1640 supplemented with $10 \% \mathrm{FCS}, 50 \mu \mathrm{M}$ 2-mercaptoethanol, $1 \mu \mathrm{M}$ pyruvate, $50 \mu \mathrm{g} / \mathrm{mL}$ gentamycin, $25 \mu \mathrm{g} / \mathrm{mL}$ GM-CSF, and $10 \mu \mathrm{g} / \mathrm{mL}$ IL-4. On day 6, DCs were harvested from the nonadherent and loosely adherent cells.

\section{Preparation of DCs fused with carcinoma cells}

Fused DCs were generated as described previously. ${ }^{33}$ Briefly, DCs were harvested at day 6. DCs were mixed with carcinoma cells treated with $10 \mathrm{mg} / \mathrm{mL}$ mitomycin C (Sigma-Aldrich) for 20 minutes at a 3:1 ratio, and were incubated in serum-free RPMI 1640 containing 50\% polyethylene glycol (SigmaAldrich) for 7 minutes. After slowly diluting with serum-free RPMI 1640, the cells were washed and resuspended in RPMI 
1640 supplemented with $10 \%$ FCS, $25 \mu \mathrm{g} / \mathrm{mL}$ GM-CSF, and $10 \mu \mathrm{g} / \mathrm{mL}$ IL-4. DCs cocultured with carcinoma cells at the same ratio in RPMI 1640 supplemented with FCS, GM-CSF, and IL-4 were used as "nonfused DCs." The next day, fused DCs and nonfused DCs were induced to mature with $1 \mu \mathrm{g} / \mathrm{mL}$ LPS for 24 hours. To determine fusion efficiency, DCs were labeled with PKH26 red fluorescent dye, and tumor cells were labeled with PKH67 green fluorescent dye prior to cell fusion. Individual cells or fused DCs were analyzed by flow cytometry and fluorescence microscopy. As a control, DCs generated from PBMCs and stimulated with LPS were examined, which were used as "DCs alone."

\section{Flow cytometry analysis}

Cells were washed with $0.01 \mathrm{M}$ phosphate-buffered saline, pH 7.4 and incubated with antibodies directed against MHC class I (W6/32; mouse IgG2aк; Dako, Glostrup, Denmark), MHC class II (HLA-DR) (mouse IgG1; Nichirei, Tokyo, Japan), CD40 (LOB-11; mouse IgG1; Santa Cruz Biotechnology, Santa Cruz, CA, USA), CD80 (MAB104; mouse IgG1; Immunotech, Marseille, France), and CD86 (BU63; mouse IgG1, Ylem, Roma, Italy) for 30 minutes on ice. After washing with phosphate-buffered saline, the cells were incubated with fluorescent isothiocyanate (FITC)-conjugated goat anti-mouse IgG (Beckman Coulter, Marseille, France) for 30 minutes on ice. In the case of FITCconjugated antibody against ligand (Beckman Coulter), cells were incubated for 30 minutes on ice. The appropriate respective mouse isotype controls (Dako) were employed. Samples were washed, fixed in 4\% paraformaldehyde, and analyzed by FACS-Calibur and CellQuest software (BD, Franklin Lakes, NJ, USA).

\section{Cytotoxicity assay}

Fused DCs, nonfused DCs, and DCs alone were cocultured with autologous nonadherent PBMCs in a 1:20 ratio, in the presence of 25 units/mL IL-2. Fifty percent of the medium was replaced on days 3 and 5, and the cultures were restimulated by freshly isolated DCs with culture medium containing 25 units/mL IL-2 on day 7. On day 14, the stimulated PBMCs were harvested and washed three times in serum-free medium and applied as effectors at various effector/target ratios. Pancreatic carcinoma cells as target cells $\left(1 \times 10^{4}\right)$ were cocultured with effector cells for 4 hours at $37^{\circ} \mathrm{C}$. To measure lactate dehydrogenase (LDH) release from targets, the supernatants were harvested and cytotoxicity was examined using the LDH Cytotoxicity Detection Kit (Takara Bio, Tokyo, Japan) as described previously. ${ }^{34}$ Briefly, $100 \mu \mathrm{L}$ of
LDH substrate was added to the 96-well flat-bottomed plate and incubated for 30 minutes at room temperature. The optical absorbance of red formazan was determined spectrophotometrically at a wavelength of $490 \mathrm{~nm}$. Spontaneous release of LDH by effectors or targets was assessed by incubation of the effectors or targets alone, respectively. Maximum release of LDH was determined by incubation of the targets in $1 \%$ Triton X-100. The percentage of specific cytotoxicity was calculated according to the following formula:

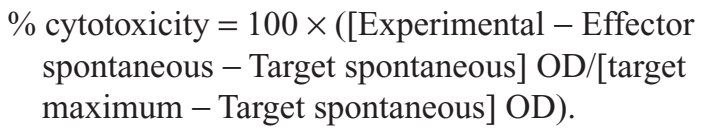

To obtain DCs and PBMCs from peripheral blood, this study was carried out in accordance with the Declaration of Helsinki and the Ethics Code for Human Experimentation, Yamagata University School of Medicine, Yamagata, Japan. Institutional review board approval was obtained, and all donors signed informed consent.

\section{Quantification of cytokine secretion}

The supernatants of the cocultures were harvested 48 hours after the second stimulation with fused DCs, and the concentrations of human cytokines, interferon- $\gamma$, IL-10 (Endogen, Rockford, IL, USA), TGF- $\beta 1$, and PGE 2 (R\&D Systems, Minneapolis, MN, USA) were quantified by enzyme-linked immunosorbent assay (ELISA). In other experiments, the supernatants of the media were harvested from 48-hourcultured respective pancreatic carcinoma cells, DCs, and fused DCs at a density of $2 \times 10^{5} / \mathrm{mL}$ in 6 -well plate, and the concentrations of human cytokines, vascular endothelial growth factor (VEGF) (Endogen), IL-10, TGF- $\beta 1$, and PGE $_{2}$ were quantified by ELISA.

\section{Reverse transcription-polymerase chain reaction (RT-PCR) for IL-2 I}

RNA was extracted from pancreatic carcinoma cells and PBMCs using RNeasy Mini Kit (Qiagen, Hilden, Germany) and was reverse-transcribed by QIAGEN OneStep RT-PCR Kit (Qiagen) according to the manufacturer's instructions. Amplification was performed in a total volume of $25 \mu \mathrm{L}$ for 35 cycles of 30 seconds at $94^{\circ} \mathrm{C}, 30$ seconds at $54^{\circ} \mathrm{C}$, and 30 seconds at $72^{\circ} \mathrm{C}$. PCR products were resolved by electrophoresis on $2 \%$ agarose gels (Funakoshi, Tokyo, Japan), the bands were visualized by ethidium bromide (Bio-Rad Laboratories, Hercules, CA, USA) staining, and photographs of the gels were taken. The positive control for the expression 
of IL-21 mRNA was RNA from normal PBMCs treated in anti-CD3-coated plates for 24 hours.

Primers used to detect IL-21 mRNA (GenBank accession number: BC066258) were 5'-ACAGACTA ACATGCCCTTCA-3' for the forward primer, and 5'-TCTTCACTTCCGTGTGTTCT-3' for the reverse primer, which produced PCR products of 134 bps. IL-21 primers were synthesized by Nihon Gene Research Laboratories (Miyagi, Japan). Primers used to detect $\beta$-actin mRNA were 5'-GATCAGCAAGCAGGAGTATG-3' for the forward primer, and 5'-GGCCATTCTCCTTAGAGAGA-3' for the reverse primer, which produced PCR products of $390 \mathrm{bps}$.

\section{Analysis of $\mathrm{CD}^{+} \mathrm{CD} 25^{\text {high }} \mathrm{T}$ cells in cocultured PBMCs}

PBMCs cocultured with fused DCs or DCs alone for 14 days were washed and incubated with a FITC-conjugated antibody against CD4 (13B8.2; mouse IgG1; Beckman Coulter) and a phycoerythrin-conjugated antibody against CD25 (B1.49.4; mouse IgG2aא; Immunotech) for 30 minutes on ice. Mouse isotype controls were employed. Cells were washed again and analyzed by flow cytometry using a live gate set around viable lymphocytes based on their forward scatter/side scatter characteristics. As a control, $\mathrm{CD} 4{ }^{+} \mathrm{CD} 25^{\text {high }} \mathrm{T}$ cells in PBMCs before coculture were analyzed.

\section{Expression of FOXP3 and IL- 10 in cocultured PBMCs}

Cocultured PBMCs were immunostained for FOXP3 to recognize $\mathrm{CD} 4^{+} \mathrm{CD} 25^{\text {high }}$ Tregs. Briefly, PBMCs cocultured with fused DCs or DCs alone for 14 days were washed, and cytospins from PBMCs were air-dried and fixed for 10 minutes in acetone. Endogenous peroxidase activity was blocked with methanol containing $0.3 \%$ hydrogen peroxide. Monoclonal antibody anti-FOXP3 (ab20034; mouse IgG1; Abcam, Cambridge, England) was incubated at $4^{\circ} \mathrm{C}$ overnight on the cytospin slides. The labeled streptavidinbiotin-peroxidase method (Ultratech HRP Streptavidinbiotin Universal Detection System; Dako) was used, and a positive reaction was detected as a brown color with 3,3'diaminobenzidine (Dojin Chemicals, Kumamoto, Japan). The slides were counterstained with 1\% methyl green (Muto Pure Chemicals, Tokyo, Japan). Positive cells were counted in at least 50 fields under a light microscope $(\times 200)$ by two observers independently. As a control, $\mathrm{FOXP}^{+} \mathrm{T}$ cells in PBMCs before coculture were analyzed, respectively. Next, phycoerythrin-conjugated antibody against IL-10 (JES3-19F1; rat IgG2a; PharMingen, San Diego, CA, USA) was incubated at $4^{\circ} \mathrm{C}$ overnight on the cytospin slides. The number of positive cells was counted in at least 50 fields under a fluorescence microscopy $(\times 200)$.

\section{Statistical analysis}

Data were expressed as the mean \pm standard deviation. Statistical significance was determined by the Student's $t$-test for paired data using StatView-software (Abacus Concepts, Berkeley, CA, USA). Differences were considered statistically significant for $P<0.05$.

\section{Results}

\section{Characterization of DCs fused with pancreatic carcinoma cells}

Fusion efficiency of DCs labeled with PKH26 and carcinoma cells labeled with PKH67 was confirmed by fluorescence microscopy. The population of fused DCs was $43.9 \% \pm 4.85 \%$ of total cells by flow cytometry. The expression of MHC class II and costimulatory molecules on DCs was then analyzed by flow cytometry. Unstimulated (immature) nonfused DCs strongly expressed MHC class II (HLA-DR) and CD40, and low levels of CD80 and CD86 (Figure 1). Nonfused DCs stimulated by LPS (mature DCs) strongly expressed MHC class II and costimulatory molecules such as CD80, CD86, and CD40. The immunophenotype of fused DCs (Panc-1 [Figure 1], KP-1NL, KP-3L, and QGP-1 [data not shown]) was similar to that of mature DCs.

\section{Induction of cytotoxicity against pancreatic carcinoma cell lines}

To assess the induction of antitumor immune response by fused DCs against the pancreatic carcinoma cell lines, fused DCs were cocultured with autologous PBMCs. As a control, PBMCs were also cocultured with nonfused DCs or DCs alone. PBMCs cocultured with DCs fused with Panc-1 (DC/ Panc-1), KP-1NL (DC/KP-1NL), or KP-3L (DC/KP-3L) induced significant cytotoxicity against tumor targets compared with those cocultured with DCs alone $(P<0.05$; Figure 2A). By contrast, PBMCs cocultured with DCs fused with QGP-1 (DC/QGP-1) induced only a low level of cytotoxicity and there was no significant difference between fused DCs and controls (nonfused and DCs alone). Furthermore, when the cytotoxicity of PBMCs cocultured with fused DCs was compared among pancreatic carcinoma cell lines, the level of cytotoxicity in DC/QGP-1 was significantly lower compared with that of other carcinoma cell lines $(P<0.05$; Figure 2B). 

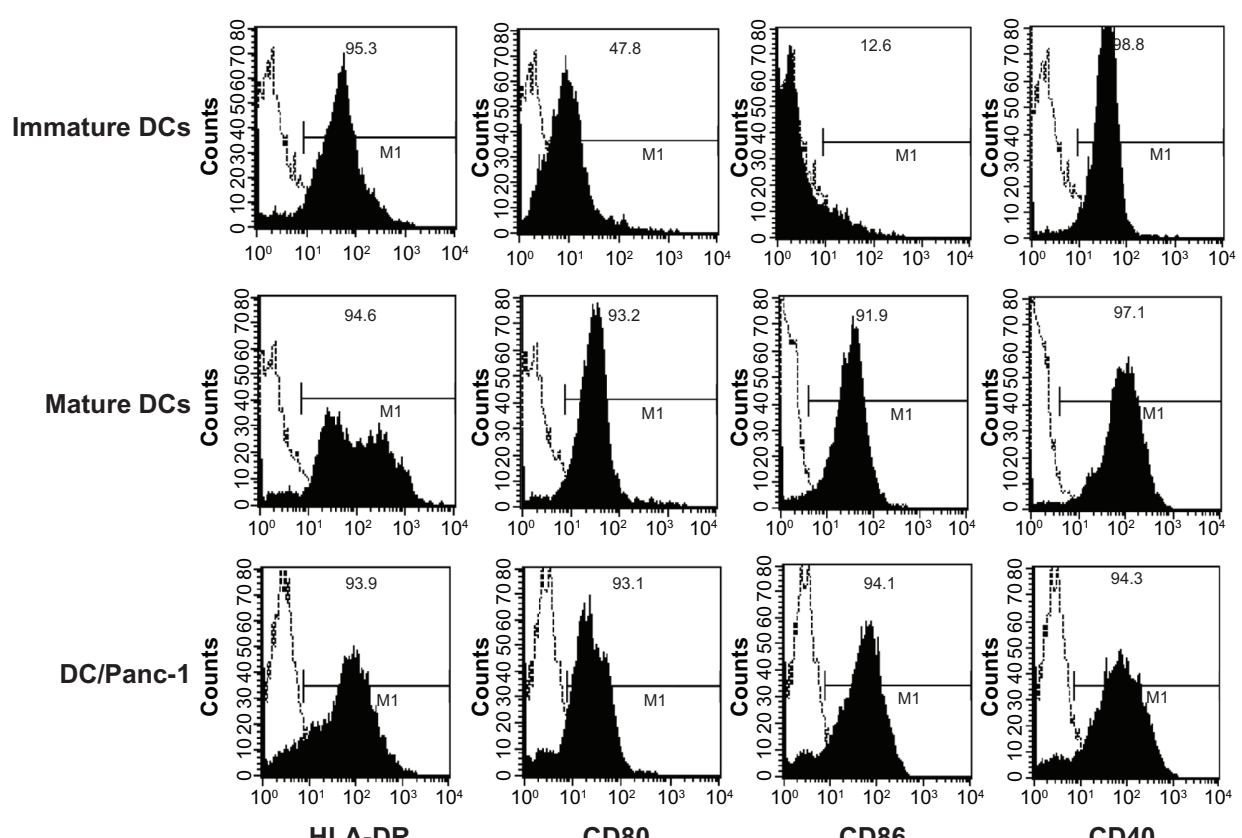

Figure I Flow cytometric characterization of dendritic cells (DCs) fused with pancreatic carcinoma cells.

Notes: Unstimulated (immature) nonfused DCs strongly expressed MHC class II (HLA-DR) and CD40, and low levels of CD80 and CD86. Nonfused DCs stimulated by LPS (mature DCs) strongly expressed MHC class II and costimulatory molecules such as CD80, CD86, and CD40. The immunophenotype of fused DCs was similar to that of mature DCs.

Abbreviation: MHC, major histocompatibility complex.

\section{Secretion of immunoregulatory cytokines from pancreatic carcinoma cell lines}

It was investigated whether or not the four carcinoma cell lines produced immunoregulatory cytokines, such as IL-10, TGF- $\beta 1$, VEGF, and PGE $_{2}$ by ELISA. IL-10 and TGF- $\beta 1$ were secreted at very low levels from the four carcinoma cell lines (Figure 3A). A greater amount of VEGF, which suppresses the differentiation and maturation of DCs, was secreted from Panc-1 and QGP-1 compared with KP-3L $(P<0.05)$. $\mathrm{PGE}_{2}$ was secreted from KP-3L at a higher level than from the other cell lines. IL-21 mRNA, that likely suppressed the maturation of DCs and failed to induce antigenspecific $\mathrm{T}$ cell proliferation, was not expressed in any tumor cells by RT-PCR (Figure 3B). Moreover, to confirm the maturation of fused DCs, the expression of MHC class II and costimulatory molecules, such as CD80, CD86, and CD40, on DC/QGP-1 or DC/KP-3L was investigated by flow cytometry. The expression of these molecules was increased, consistent with the immunophenotype of mature DCs, and no suppression of the maturation of fused DCs was observed.

\section{Secretion of immunoregulatory cytokines from PBMCs cocultured with fused DCs}

ELISA was performed to assess interferon- $\gamma$, IL-10, TGF- $\beta 1$, and $\mathrm{PGE}_{2}$ secretion in the supernatants of PBMCs 48 hours after the second stimulation by DCs fused with QGP-1, as a cell line that induced a low level of cytotoxicity, or DCs fused with KP-3L, as a cell line that induced a high level of cytotoxicity. As a control, cytokines in the supernatants of PBMCs cocultured with DCs alone were assessed. Interferon- $\gamma$ secretion in PBMCs cocultured with DC/QGP-1 or DC/KP-3L was significantly higher than that in DCs alone ( $P<0.05$; Figure 4$)$. There was no significant difference between DC/QGP-1 and DC/KP-3L. IL-10 secretion in PBMCs cocultured with DC/ QGP-1 was significantly higher than that with DC/KP-3L and DCs alone $(P<0.05)$. The level of TGF- $\beta 1$ secretion was not significantly different among the three experimental groups. $\mathrm{PGE}_{2}$ secretion in the PBMCs cocultured with DC/QGP-1 or DC/KP-3L was increased significantly, compared with that with DCs alone. There was no significant difference between DC/QGP-1 and DC/KP-3L. To investigate whether PBMCs cocultured with DC/QGP-1 secreted IL-10, cocultured PBMCs were immunofluorescently stained with anti-IL-10 antibody. The number of IL- $10^{+}$cells in PBMCs cocultured with DC/ QGP-1 was significantly increased compared with that with DC/KP-3L and DCs alone $(P<0.05$; Figure 5$)$.

\section{Expansion of $C D 4^{+} \mathrm{CD} 25^{\text {high }}$ Tregs}

The relative expansion of Tregs in PBMCs cocultured with DC/QGP-1 and DC/KP-3L for 14 days was compared 

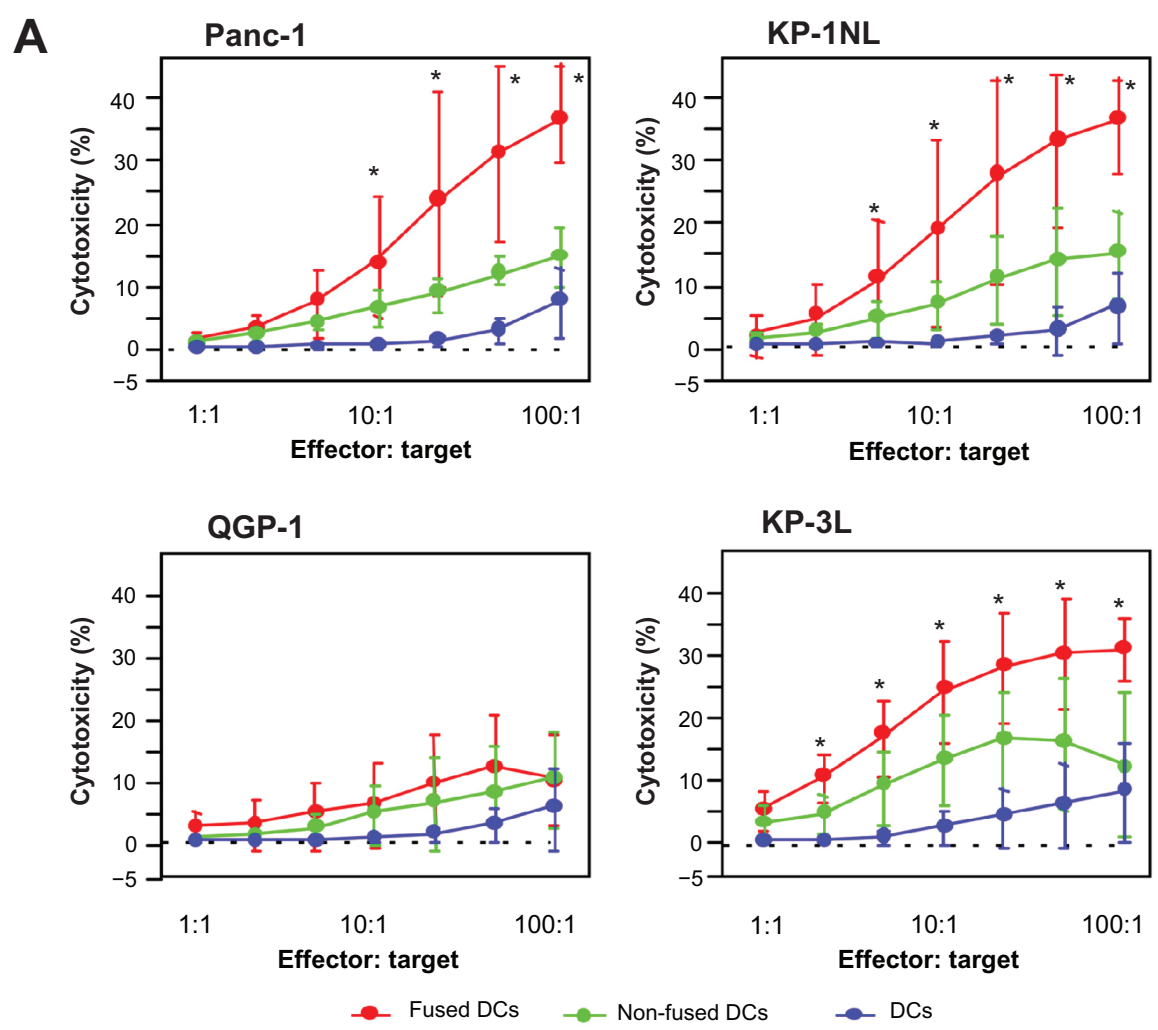

B

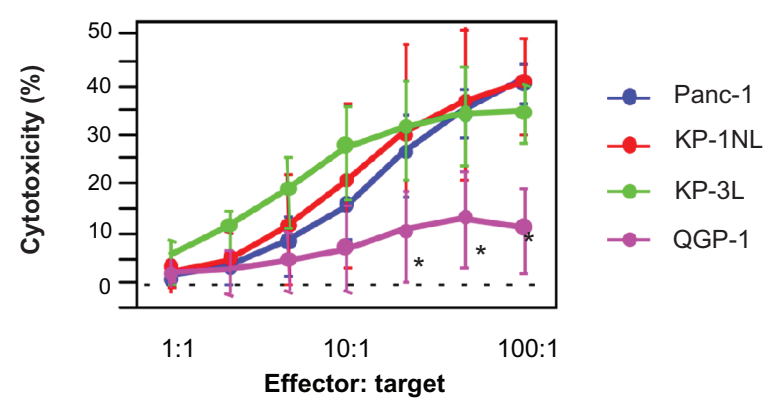

Figure 2 Cytotoxicity against the respective tumor target of peripheral blood mononuclear cells (PBMCs) cocultured with dendritic cells (DCs) fused with pancreatic carcinoma cells, such as Panc-I, KP-INL, QGP-I, and KP-3L. PBMCs were harvested from the cocultures with fused DCs, nonfused DCs, and DCs alone. Cytotoxicity induced by PBMCs was analyzed to use with lactate dehydrogenase (LDH) cytotoxicity detection kit. (A) Cytotoxicity of PBMCs cocultured with fused DCs, nonfused DCs, and DCs alone. (B) Cytotoxicity of PBMCs cocultured with fused DCs compared among pancreatic carcinoma cell lines. (B) Is an amalgam of separate components of (A). Notes: Results are expressed as the mean \pm standard deviation (SD) of four independent experiments, and compared by Student's $t$-test. $* P<0.05$.

with that in the PBMCs before coculture. $\mathrm{CD} 4{ }^{+} \mathrm{CD} 25^{\text {high }}$ Tregs in PBMCs cocultured with DC/QGP-1 were significantly increased compared with those in PBMCs cocultured with DC/KP-3L and DCs alone by flow cytometry $\left(\mathrm{CD} 4^{+} \mathrm{CD} 25^{\text {high }}\right)$ (Figure 6) and immunocytochemistry (FOXP3) (Figure 7).

\section{Expression of MHC class I in pancreatic carcinoma cell lines}

The expression of MHC class I in carcinoma cell lines was evaluated by flow cytometry. Almost all tumor cells in the cell lines were positive for MHC class I antigen (Figure 8A) and there was no significant difference among cell lines. By contrast, from the perspective of mean fluorescent intensity, MHC class I antigen in KP-1NL and QGP-1 was significantly lower compared with that in KP-3L $(P<0.05$; Figure 8B).

\section{Expression of Fas ligand in pancreatic carcinoma cells}

Fas ligand was not detectable in carcinoma cells by flow cytometry (data not shown). 


\section{A}

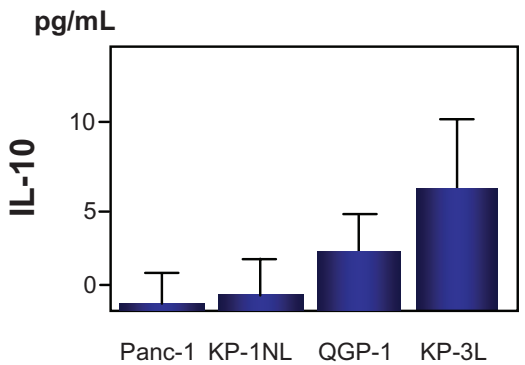

$\mathrm{pg} / \mathrm{mL}$

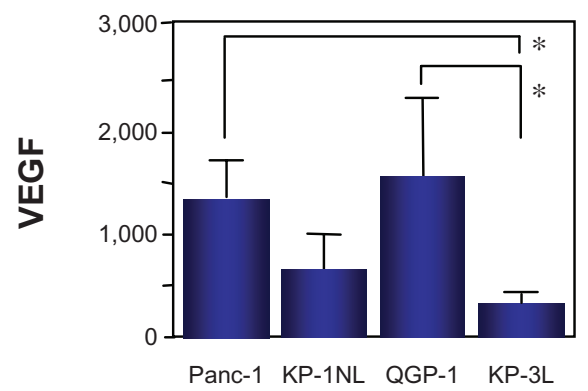

$\mathrm{pg} / \mathrm{mL}$

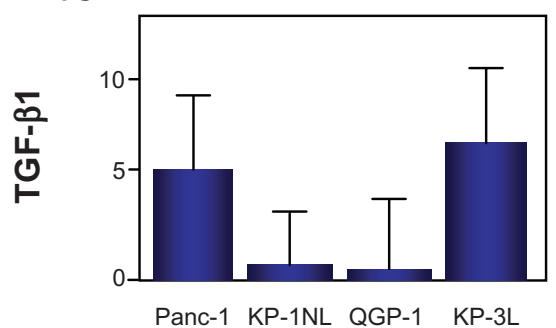

Panc-1 KP-1NL QGP-1 KP-3L

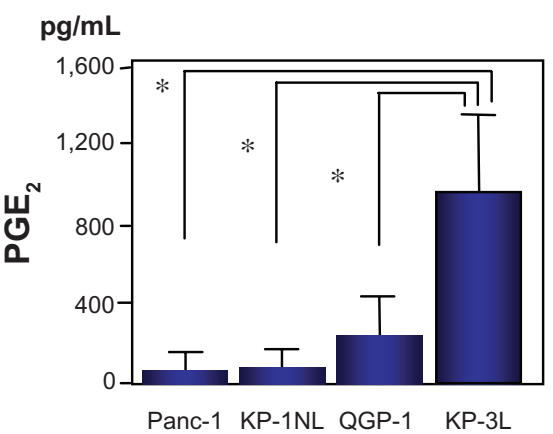

B

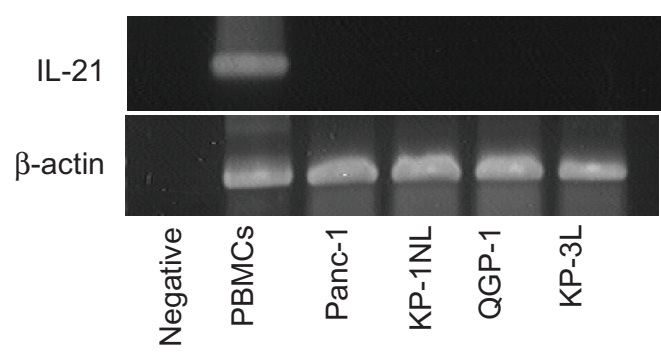

Figure 3 (A) Immunoregulatory cytokine secretion from pancreatic carcinoma cell lines and dendritic cells (DCs). Forty-eight hours after suspending carcinoma cells and DCs at a density of $2 \times 105 / \mathrm{mL}$ in the 6-well plate, the supernatants of the medium were harvested, and quantified for secreted interleukin-I0 (IL-I0), transforming growth factor (TGF)- $\beta$ I, vascular endothelial growth factor (VEGF), and prostaglandin $E_{2}\left(P G E_{2}\right)$ by enzyme linked immunosorbent assay (ELISA). Results are expressed as the mean \pm standard deviation (SD) of four independent experiments, and compared by Student's $t$-test. $* P<0.05$. (B) IL-2I mRNA by reverse transcription-polymerase chain reaction (RT-PCR). Notes: The expression level of IL-2I mRNA was assessed in four pancreatic carcinoma cell lines. The positive control for the expression of IL-2I mRNA was RNA from normal peripheral blood mononuclear cells (PBMCs) treated in anti-CD3-coated plates for 24 hours. The result is representative of three independent experiments.

\section{Discussion}

The current study firstly investigated whether DCs fused with pancreatic carcinoma cells induced cytotoxicity against the tumor targets in four human pancreatic carcinoma cell lines, and examined the contribution of immunosuppressive factors against the cytotoxicity of PBMCs cocultured with fused DCs among cell lines.

This study demonstrated that DC/Panc-1, DC/KP-1NL, and $\mathrm{DC} / \mathrm{KP}-3 \mathrm{~L}$ significantly induced cytotoxicity against the tumor targets compared with DCs alone, excluding DC/ QGP-1. When the cytotoxicity induced by fused DCs was compared among the pancreatic carcinoma cell lines, the level of cytotoxicity in QGP-1 was significantly lower compared with that in the other cell lines.
Recent studies have demonstrated that the mechanisms of tumor escape from immune recognition/destruction are likely to be multifactorial, such the downregulation of MHC class I molecules, loss of tumor antigens, defective death receptor signaling, production of immunosuppressive cytokines, and the existence of suppressive cells. ${ }^{18}$ To reveal the factors that suppressed cytotoxicity of PBMCs cocultured with fused DCs, we examined these factors among pancreatic carcinoma cell lines and demonstrated that significantly higher levels of VEGF were detected in the supernatants of Panc-1 and QGP-1 compared with KP-3L. The previous study indicated that caudal related homeobox gene 2 (CDX2), an intestinespecific tumor suppressor gene, was expressed at a high level in QGP-1 cells, and cell proliferation and cyclin D1 mRNA 

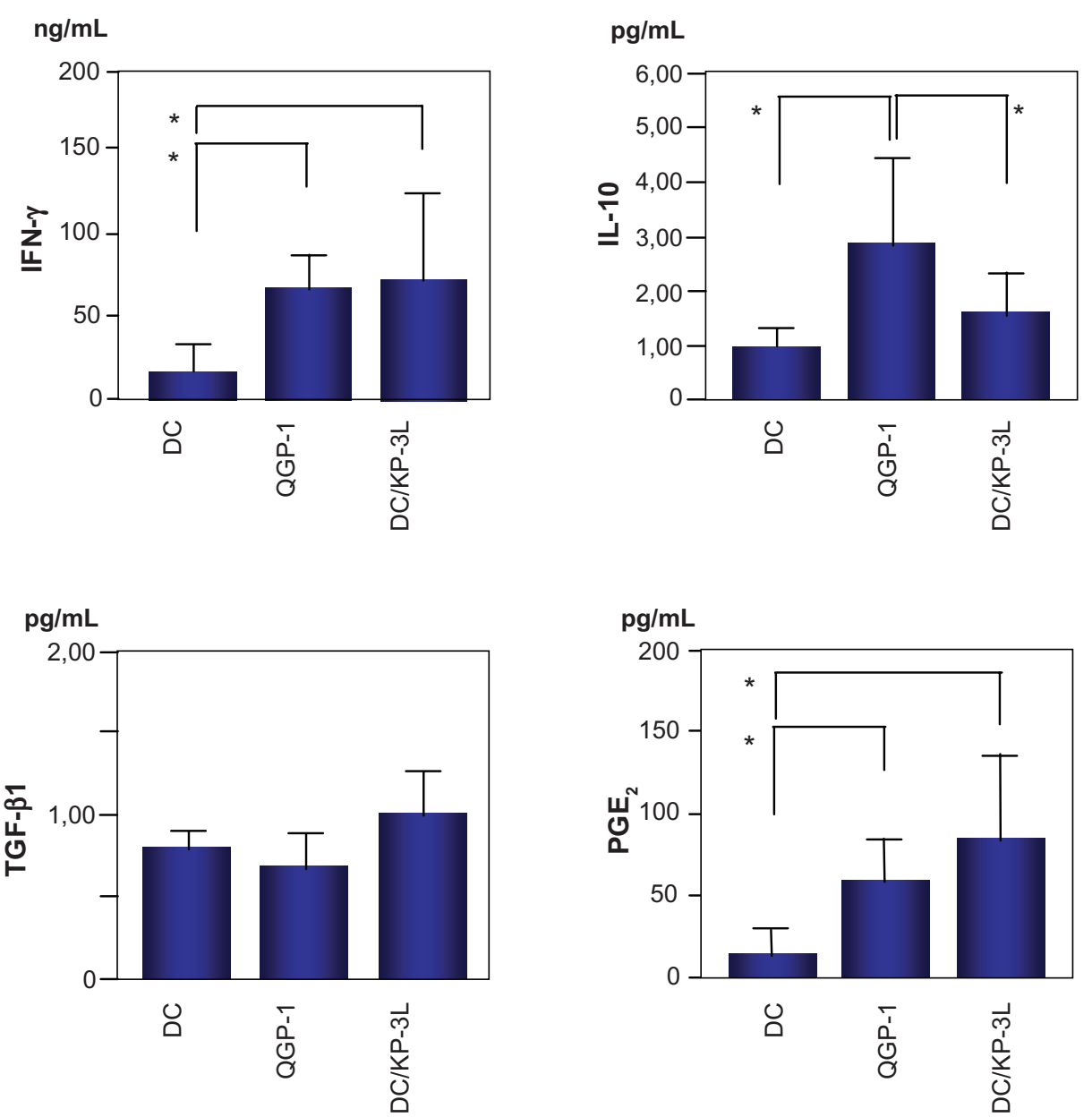

Figure 4 Immunoregulatory cytokine secretion from peripheral blood mononuclear cells (PBMCs) cocultured with dendritic cells (DCs) fused with QGP-I (DC/QGP-I), DCs fused with KP-3L (DC/KP-3L), or DCs alone.

Notes: Forty-eight hours after the second stimulation with fused DCs or DCs alone, the supernatants of PBMCs were harvested and quantified for secreted interferon (IFN)- $\gamma$, interleukin- 10 (IL-I0), transforming growth factor (TGF)- $\beta$ I, and prostaglandin $\mathrm{E}_{2}$ (PGE $)$ by enzyme linked immunosorbent assay (ELISA). Results are expressed as the mean \pm standard deviation (SD) of six independent experiments, and compared by Student's $t$-test. $* P<0.05$.

level were inhibited significantly after CDX2 transfection in pancreatic cancer cells, suggesting that CDX2 might play a role in inhibiting cell proliferation and repressing cyclin D1 transcriptional activity. ${ }^{35}$ However, in our study there were not any significant differences in induction of cytotoxicity among DCs alone, nonfused DCs, and fused DCs (DC/QGP1) (Figure 2). Thus, the immunosuppressive potential of DC/ QGP-1 may be due to only "active" in the context of professional antigen presentation from immune cells rather than an intrinsic property of QGP-1. VEGF is produced by many tumors and is important not only for tumor vascularization, but is also a key factor produced by solid tumors to inhibit immune recognition and to prevent DC differentiation and maturation. ${ }^{36}$ Furthermore, to examine whether increased production of VEGF was associated with the suppression of cytotoxicity against pancreatic carcinoma cell lines, this study investigated the expression of MHC class II and costimulatory molecules in DC/QGP-1 or DC/KP-3L by flow cytometry. The expression of these molecules was similar to the expression of surface antigens in mature DCs. DC/QGP-1 and $\mathrm{DC} / \mathrm{KP}-3 \mathrm{~L}$ matured sufficiently to induce the immune response. Independently, expression of MHC class I antigens in KP-1NL and QGP-1 was significantly downregulated compared with KP-3L, when these results were expressed as fluorescent intensity. Downregulation of MHC class I was recognized in QGP-1 as well as KP-1NL, which induced a higher degree of cytotoxicity. MHC class I molecule downregulation occurs frequently in many cancers, and the altered phenotypes of MHC class I antigen expression can allow tumors to avoid recognition or survive attack by cytotoxic $\mathrm{CD}^{+}$T-cells. ${ }^{37}$ Kasuya et al have also demonstrated that the hypervascular pancreatic tumor, QGP-1 secreted a higher level of VEGF under a hypoxic environment than the hypovascular pancreatic ductal cell lines (BxPC-3 and AsPC-1). ${ }^{38}$ 

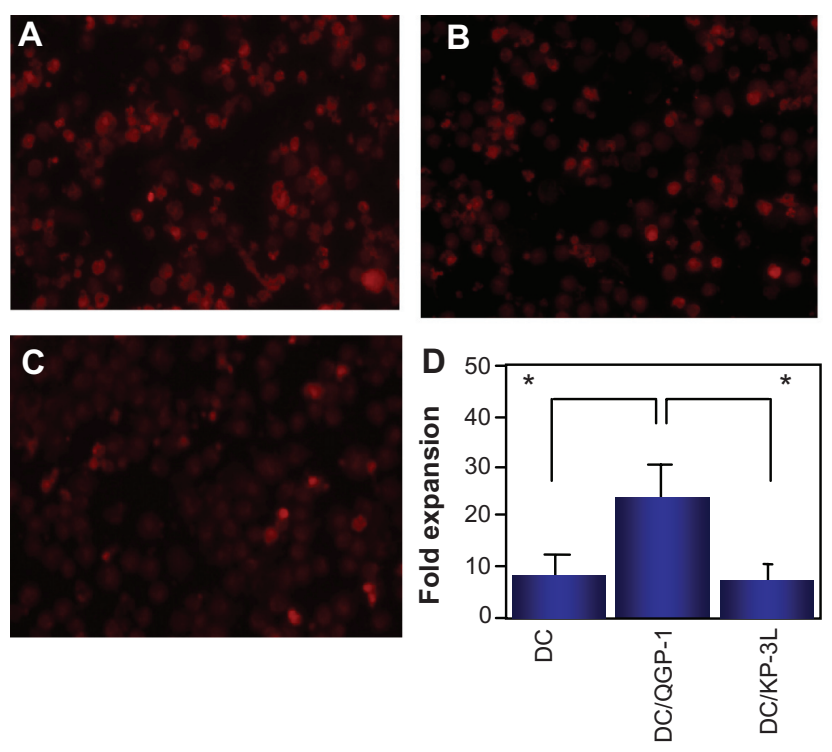

Figure 5 The induction of interleukin-10 (IL-10+) cells in the peripheral blood mononuclear cells (PBMCs) cocultured with dendritic cells (DCs). IL- $10^{+}$cells in PBMCs cocultured with DCs fused with QGP-I (DC/QGP-I) (A), KP-3L (DC/ $\mathrm{KP}-3 \mathrm{~L})(\mathbf{B})$, or DCs alone $(\mathbf{C})(\times 200)$. The number of positive cells was counted in at least 50 fields under a light microscopy. The number of $\mathrm{IL}-10^{+}$cells after coculture with DC/QGP-I was increased. Results are expressed as the mean \pm standard deviation (SD) of five independent experiments (D).

These findings did not demonstrate that high secretion of VEGF and downregulation of MHC class I could be related to the immunosuppression of DC/QGP-1, despite a significant difference between QGP-1 and KP-3L. Furthermore, there was the elevated cytotoxicity of the nonfused DCs/tumor cell mixture (Figure 2). Nonfused DCs/tumor cell mixture may recognize a low level of soluble antigens secreted from

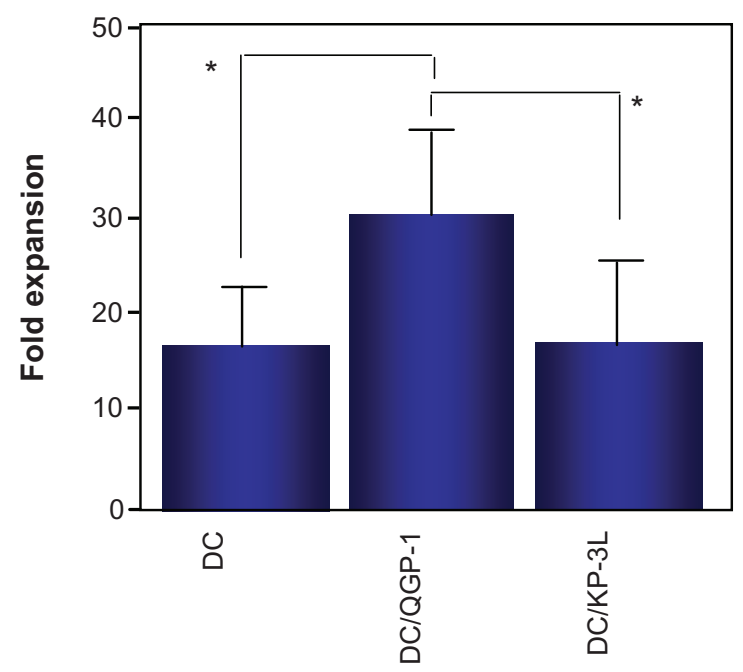

Figure $6 \mathrm{CD}^{+} \mathrm{CD} 25^{\text {high }} \mathrm{T}$ cells in peripheral blood mononuclear cells cocultured with DC/QGP-I were significantly increased compared with those in peripheral blood mononuclear cells cocultured with DC/KP-3L and DCs alone.

Notes: Results are expressed as the mean \pm standard deviation (SD) of five independent experiments. $* p<0.05$. tumor cells or surface antigens expressed weakly on the cell surface of tumor cells.

Recent studies have reported that $\mathrm{CD} 4{ }^{+} \mathrm{CD} 25^{\text {high }}$ Tregs are immunoregulatory and are important in immunological tolerance to self-antigens. ${ }^{19-22}$ The present study demonstrated that $\mathrm{CD} 4^{+} \mathrm{CD} 25^{\text {high }}$ Tregs in PBMCs cocultured with DC/QGP-1 were significantly increased compared with those in PBMCs cocultured with DC/KP-3L or DCs alone by flow cytometry $\left(\mathrm{CD} 4{ }^{+} \mathrm{CD} 25^{\text {high }}\right)$ and immunocytochemistry (FOXP3). To investigate whether Tregs induced an immunosuppressive cytokine profile, the secretion of IL-10 and TGF- $\beta 1$ was subsequently analyzed in the supernatants of PBMCs cocultured with fused DCs. There was no significant difference in the level of TGF- $\beta 1$ secretion among the three experimental conditions. Although a recent study demonstrated the induction of Treg function in $\mathrm{CD}^{+} \mathrm{T}$ cells by $\mathrm{PGE}_{2}$ secreted from carcinoma cells and the present study revealed that $\mathrm{PGE}_{2}$ was secreted to a much higher level in the supernatants of KP-3L but not QGP-1 in the supernatants of PBMCs cocultured with DC/QGP-1 or DC/KP-3L compared with that in DCs alone, there was no significant difference between DC/QGP-1 and DC/KP-3L. Cytokines (IL-10 and TGF- $\beta$ ), immature myeloid DCs, CpG-ODN stimulated plasmacytoid DCs, and immunomodulatory drugs (vitamin D3) can induce $\mathrm{CD} 4{ }^{+} \mathrm{CD} 25^{-} \mathrm{T}$-cells to differentiate into $\mathrm{CD} 4{ }^{+} \mathrm{CD} 25^{+}$ T cells. ${ }^{19-22,39}$ In this study, IL-10 secretion in PBMCs cocultured with DC/QGP-1 was significantly higher than that with $\mathrm{DC} / \mathrm{KP}-3 \mathrm{~L}$ or DCs alone, suggesting that the source of increasing IL-10 may include DCs functionally altered by fusion mechanism and IL-10 secreting Tregs such as $\mathrm{CD} 4{ }^{+} \mathrm{CD} 25^{\text {high }} \mathrm{T}$ cells and $\mathrm{CD} 4{ }^{+}$type 1 Tregs. ${ }^{40}$ Subsequently, increasing IL-10 may be related to the expansion of Tregs and the induction of the immunosuppressive response. Leao et $\mathrm{al}^{24}$ demonstrated that effective depletion of Treg cells allows the recruitment of mesothelin-specific $\mathrm{CD} 8^{+} \mathrm{T}$ cells to the antitumor immune response against a mesothelin-expressing mouse pancreatic adenocarcinoma. Yamamoto et $\mathrm{al}^{41}$ also reported that Treg depletion combined with DC/tumor cell fusion hybrid vaccine enhanced the efficacy of immunotherapy in pancreatic cancer by activating CTLs. Similar effectiveness of Treg depletion in DC vaccination has been indicated in eradication of lymphoma cells in mice. ${ }^{42}$

Although the overall 5-year survival for patients of pancreatic adenocarcinoma is only $1 \%-4 \%$, that of malignant pancreatic endocrine carcinoma was $49 \% .{ }^{43}$ DC-based vaccination against pancreatic endocrine carcinoma with liver metastases revealed a decreased tumor marker level and a tumor regression of metastases. ${ }^{44}$ However, information 

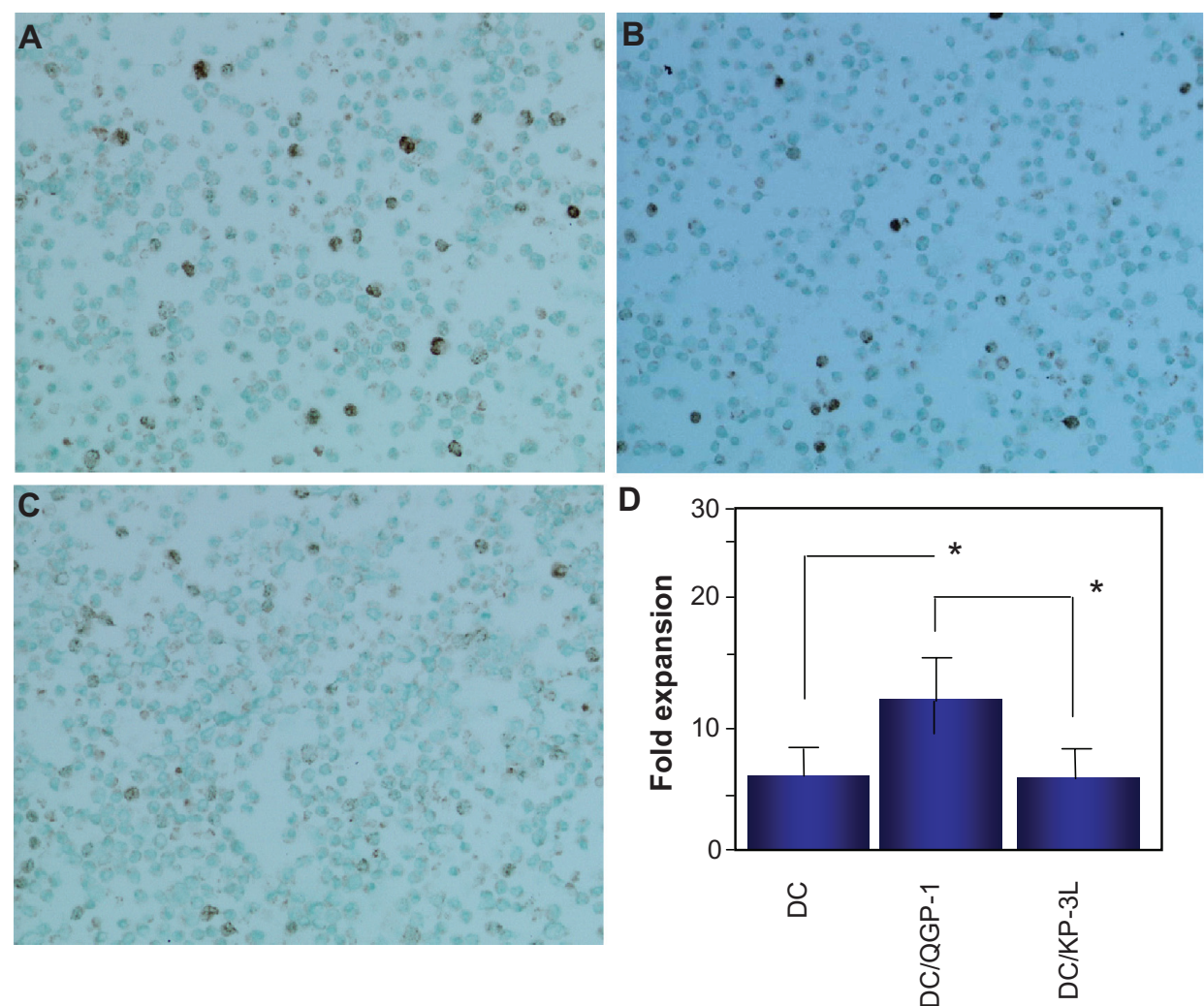

Figure 7 The induction of FOXP3 $3^{+}$cells in the peripheral blood mononuclear cells (PBMCs) cocultured with dendritic cells (DCs). FOXP3 $3^{+}$cells in PBMCs cocultured with dendritic cells (DCs) fused with QGP-I (DC/QGP-I) (A), DCs fused with KP-3L (DC/KP-3L) (B), or DCs alone (C) $(\times 200)$. The number of positive cells was counted in at least 50 fields under a light. FOXP3 ${ }^{+}$cells in PBMCs cocultured with DC/QGP-I were significantly increased compared with those in PBMCs cocultured with DC/KP-3L and DCs alone $(* P<0.05)$. Results are expressed as the mean \pm standard deviation $(S D)$ of five independent experiments (D).

about effects of DC-based immunotherapy against pancreatic endocrine carcinoma is quite limited. Thus, it is critical to compare the difference in induction of cytotoxicity between pancreatic ductal carcinoma and endocrine carcinoma, as shown in this study.

In conclusion, the present study firstly demonstrated that there were significant differences in the induction of CTL and
A

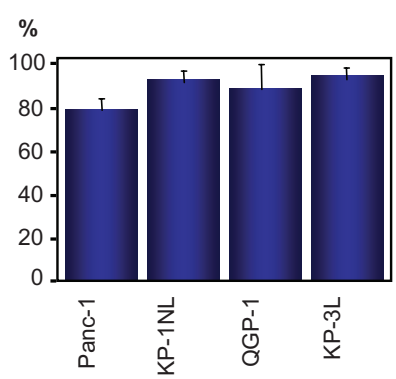

B

MFI

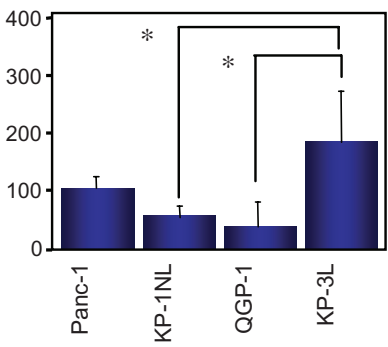

Figure 8 Expression of major histocompatibility complex (MHC) class I in pancreatic carcinoma cell lines by flow cytometry. Results are expressed as percentage of positive cells (A), or as mean fluorescence intensity (MFI) (B).

Notes: Results are expressed as the mean \pm standard deviation (SD) of three independent experiments, and compared by Student's $t$-test. $* P<0.05$.
$\mathrm{CD} 4^{+} \mathrm{CD} 25^{\text {high }}$ Tregs, and the amount of IL-10 among DCs fused with different pancreatic carcinomas. These findings suggest that the reduced cytotoxicity of DC/QGP-1 may be related to the increased secretion of IL-10 and the extensive induction of $\mathrm{CD} 4{ }^{+} \mathrm{CD} 25^{\text {high }}$ Tregs.

\section{Acknowledgments}

We thank the Yamagata Red Cross Blood Center (Yamagata, Japan) for providing the buffy coat of peripheral blood samples. We also thank Mr Suzuki H and Ms Abe K of the Department of Pathology for their technical support, and Dr Okumoto K of the Department of Internal Medicine for providing $\beta$-actin primers in Yamagata University School of Medicine.

\section{Disclosure}

The authors report no conflicts of interest in this work.

\section{References}

1. Stift A, Friedl J, Dubsky P, et al. Dendritic cell-based vaccination in solid cancer. J Clin Oncol. 2003;21(1):135-142.

2. Nakamura M, Wada J, Suzuki H, Tanaka M, Katano M, Morisaki T. Long-term outcome of immunotherapy for patients with refractory pancreatic cancer. Anticancer Res. 2009;29(3):831-836. 
3. Suso EM, Dueland S, Rasmussen AM, et al. hTERT mRNA dendritic cell vaccination: complete response in a pancreatic cancer patient associated with response against several hTERT epitopes. Cancer Immunol Immunother. 2011;60(6):809-818.

4. Bauer C, Dauer M, Saraj S, et al. Dendritic cell-based vaccination of patients with advanced pancreatic carcinoma: results of a pilot study. Cancer Immunol Immunother. 2011;60(8):1097-1107.

5. Nakamura I, Kanazawa M, Sato Y, et al. Clinical evaluation of dendritic cells vaccination for advanced cancer patients at Fukushima Medica University. Fukushima J Med Sci. 2012;58(1):40-48.

6. Pecher G, Häring A, Kaiser L, Thiel E. Mucin gene (MUC1) transfected dendritic cells as vaccine: results of a phase I/II clinical trial. Cancer Immunol Immunother. 2002;51(11-12):669-673.

7. Nagaraj S, Ziske C, Strehl J, Messmer D, Sauerbruch T, Schmidt-WolfIG. Dendritic cells pulsed with alpha-galactosylceramide induce anti-tumor immunity against pancreatic cancer in vivo. Int Immunol. 2006;18(8): 1279-1283.

8. Nagaraj S, Neumann J, Winzen B, et al. Pancreas carcinoma antigen fused to invariant chain elicits T-cell response and tumor growth inhibition. Pancreas. 2008;37(3):321-327.

9. Lepisto AJ, Moser AJ, Zeh H, et al. A phase I/II study of a MUC1 peptide pulsed autologous dendritic cell vaccine as adjuvant therapy in patients with resected pancreatic and biliary tumors. Cancer Ther. 2008;6(B):955-964.

10. Béraud E, Collignon A, Franceschi C, Olive D, Lombardo D, Mas E. Investigation of a new tumor-associated glycosylated antigen as target for dendritic cell vaccination in pancreatic cancer. Oncoimmunology. 2012;1(1):56-61.

11. Hirooka Y, Itoh A, Kawashima H, et al. A combination therapy of gemcitabine with immunotherapy for patients with inoperable locally advanced pancreatic cancer. Pancreas. 2009;38(3):e69-e74.

12. Bauer C, Bauernfeind F, Sterzik A, et al. Dendritic cell-based vaccination combined with gemcitabine increases survival in a murine pancreatic carcinoma model. Gut. 2007;56(9):1275-1282.

13. Dauer M, Herten J, Bauer C, et al. Chemosensitization of pancreatic carcinoma cells to enhance T cell-mediated cytotoxicity induced by tumor lysate-pulsed dendritic cells. J Immunother. 2005;28(4):332-342.

14. Banchereau J, Palucka AK, Dhodapkar M, et al. Immune and clinical responses in patients with metastatic melanoma to $\mathrm{CD} 34^{+}$progenitorderived dendritic cell vaccine. Cancer Res. 2001;61(17): 6451-6458.

15. Boghossian S, Robinson S, Von Delwig A, Manas D, White S. Immunotherapy for treating metastatic colorectal cancer. Surg Oncol. 2012;21(2):67-77.

16. Avigan D, Rosenblatt J, Kufe D. Dendritic/tumor fusion cells as cancer vaccines. Semin Oncol. 2012;39(3):287-295.

17. Xu F, Ye YJ, Liu W, Kong M, He Y, Wang S. Dendritic cell/tumor hybrids enhances therapeutic efficacy against colorectal cancer liver metastasis in SCID mice. Scand J Gastroenterol. 2010;45(6):707-713.

18. Sadun RE, Sachsman SM, Chen X, et al. Immune signatures of murine and human cancers reveal unique mechanisms of tumor escape and new targets for cancer immunotherapy. Clin Cancer Res. 2007;13(13): 4016-4025.

19. Le DT, Jaffee EM. Regulatory T-cell modulation using cyclophosphamide in vaccine approaches: a current perspective. Cancer Res. 2012;72(14):3439-3444.

20. Schmidt A, Oberle N, Krammer PH. Molecular mechanisms of tregmediated T cell suppression. Front Immunol. 2012;3:51.

21. Raynor J, Lages CS, Shehata H, Hildeman DA, Chougnet CA. Homeostasis and function of regulatory T cells in aging. Curr Opin Immunol. 2012;24(4):482-487.

22. Facciabene A, Motz GT, Coukos G. T-regulatory cells: key players in tumor immune escape and angiogenesis. Cancer Res. 2012;72(9): 2162-2171.

23. Baratelli F, Lin Y, Zhu L, et al. Prostaglandin $\mathrm{E}_{2}$ induces FOXP3 gene expression and $\mathrm{T}$ regulatory cell function in human $\mathrm{CD}^{+} \mathrm{T}$ cells. J Immunol. 2005;175(3):1483-1490.

24. Leao IC, Ganesan P, Armstrong TD, Jaffee EM. Effective depletion of regulatory $\mathrm{T}$ cells allows the recruitment of mesothelin-specific CD8 $\mathrm{T}$ cells to the antitumor immune response against a mesothelinexpressing mouse pancreatic adenocarcinoma. Clin Transl Sci. 2008; 1(3):228-239.
25. Ziske C, Etzrodt PE, Eliu AS, et al. Increase of in vivo antitumoral activity by CD40L (CD154) gene transfer into pancreatic tumor celldendritic cell hybrids. Pancreas. 2009;38(7):758-765.

26. Nagaraj S, Neumann J, Winzen B, et al. Pancreas carcinoma antigen fused to invariant chain elicits T-cell response and tumor growth inhibition. Pancreas. 2008;37(3):321-327.

27. Nagaraj S, Ziske C, Strehl J, Messmer D, Sauerbruch T, Schmidt-Wolf IG. Dendritic cells pulsed with alpha-galactosylceramide induce anti-tumor immunity against pancreatic cancer in vivo. Int Immunol. 2006;18(8): 1279-1283.

28. Madden ME, Sarras MP Jr. Morphological and biochemical characterization of a human pancreatic ductal cell line (PANC-1). Pancreas. 1988;3(5):512-528.

29. Funakoshi A, Kono A. Growth inhibition of human pancreatic cancer cells by cholecystokinin receptor antagonist in tissue culture and in nude mice. Gastroenterol Jpn. 1992;27(1):78-82.

30. Kaku M, Nishiyama T, Yagawa K, Abe M. Establishment of a carcinoembryonic antigen-producing cell line from human pancreatic carcinoma. Gann. 1980;71(5):596-601.

31. Ikeda Y, Ezaki M, Hayashi I, Yasuda D, Nakayama K, Kono A. Establishment and characterization of human pancreatic cancer cell lines in tissue culture and in nude mice. Jpn J Cancer Res. 1990;81(10): 987-993.

32. Ramos RN, Chin LS, Dos Santos AP, Bergami-Santos PC, Laginha F, Barbuto JA. Monocyte-derived dendritic cells from breast cancer patients are biased to induce $\mathrm{CD} 4^{+} \mathrm{CD} 25^{+}$Foxp $3^{+}$regulatory T cells. J Leukoc Biol. 2012;92(3):673-682.

33. Gong J, Nikrui N, Chen D, et al. Fusions of human ovarian carcinoma cells autologous or allogenic dendritic cells induce antitumor immunity. J Immunol. 2000;165(3):1705-1711.

34. Iwashita Y, Goto S, Tominaga M, et al. Dendritic cell immunotherapy with poly(D,L-2,4-diaminobutyric acid)-mediated intratumoral delivery of the interleukin-12 gene suppresses tumor growth significantly. Cancer Sci. 2005;95(5):303-307.

35. Takahashi K, Hirano F, Matsumoto K, Aso K, Haneda M. Homeobox gene CDX2 inhibits human pancreatic cancer cell proliferation by downregulating cyclin D1 transcriptional activity. Pancreas. 2009;38(1): 49-57.

36. Sheng KC, Wright MD, Apostolopoulos V. Inflammatory mediators hold the key to dendritic cell suppression and tumor progression. Curr Med Chem. 2011;18(36):5507-5518.

37. Bernal M, Ruiz-Cabello F, Concha A, Paschen A, Garrido F. Implication of the $\beta 2$-microglobulin gene in the generation of tumor escape phenotypes. Cancer Immunol Immunother. 2012;61(9):1359-1371.

38. Kasuya K, Nagakawa Y, Suzuki M, et al. Anti-vascular endothelial growth factor antibody single therapy for pancreatic neuroendocrine carcinoma exhibits a marked tumor growth-inhibitory effect. Exp Ther Med. 2011;2(6):1047-1052.

39. Urry Z, Chambers ES, Xystrakis E, et al. The role of $1 \alpha, 25$ dihydroxyvitamin D3 and cytokines in the promotion of distinct Foxp $3^{+}$ and IL-10+CD4+ T cells. Eur J Immunol. 2012;42(10):2697-2708.

40. Levings MK, Gregori S, Tresoldi E, Cazzaniga S, Bonini C, Roncarolo MG. Differentiation of $\operatorname{Tr} 1$ cells by immature dendritic cells requires IL-10 but not CD25 ${ }^{+}$CD4 ${ }^{+}$Tr cells. Blood. 2005;105(3):1162-1169.

41. Yamamoto M, Kamigaki T, Yamashita K, et al. Enhancement of antitumor immunity by high levels of Th1 and Th17 with a combination of dendritic cell fusion hybrids and regulatory $\mathrm{T}$ cell depletion in pancreatic cancer. Oncol Rep. 2009;22(2):337-343.

42. Maksimow M, Miiluniemi M, Marttila-Ichihara F, Jalkanen S, Hänninen A. Antigen targeting to endosomal pathway in dendritic cell vaccination activates regulatory $\mathrm{T}$ cells and attenuates tumor immunity. Blood. 2006;108(4):1298-1305.

43. Ehehalt F, Saeger HD, Schmidt CM, Grützmann R. Neuroendocrine tumors of the pancreas. Oncologist. 2009;14(5):456-467.

44. Schott M, Feldkamp J, Lettmann M, Simon D, Scherbaum WA, Seissler J. Dendritic cell immunotherapy in a neuroendocrine pancreas carcinoma. Clin Endocrinol. 2001;55(2):271-277. 


\section{Publish your work in this journal}

OncoTargets and Therapy is an international, peer-reviewed, open access journal focusing on the pathological basis of all cancers, potential targets for therapy and treatment protocols employed to improve the management of cancer patients. The journal also focuses on the impact of management programs and new therapeutic agents and protocols on

patient perspectives such as quality of life, adherence and satisfaction. The manuscript management system is completely online and includes a very quick and fair peer-review system, which is all easy to use. Visit http://www.dovepress.com/testimonials.php to read real quotes from published authors.

Submit your manuscript here: http://www.dovepress.com/oncotargets-and-therapy-journal 